\title{
Research and Application of Carrier Aggregation Technology in Smart Grid
}

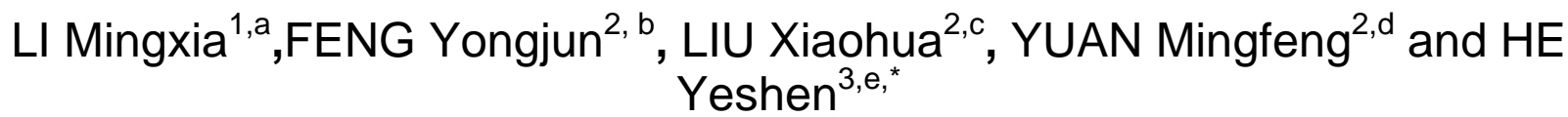 \\ ${ }^{1}$ State Grid Xinjiang Electric Power Corporation Electric Power Research Institute, Xinjiang China \\ ${ }^{2}$ State Grid Xinjiang Electric Power Corporation Marketing Department, Xinjiang China \\ ${ }^{3}$ State Grid Information \& Telecommunication Group

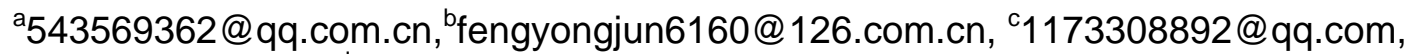 \\ dymf12@126.com, ${ }^{\mathrm{d}}$ nelsonhonger@189.cn*l
}

Keywords: Carrier Aggregation, Smart Grid, TD-LTE, Spectrum Effectiveness.

\begin{abstract}
Due to limitation of the optical fiber communication network coverage, the wireless technology is usually adapted to meet the requirement of applications in the smart grid transmission and distribution. In this paper, we study the electrical communication with integrated access technology to establish communication with the whole business model of electricity based on the same frequency and carrier aggregation TD-LTE230 MHz power wireless broadband system. We divided the power $230 \mathrm{MHz}$ private spectrum into the 40 discrete frequency bands by the carrier aggregation (CA) technology. Meanwhile, we present a novel scheme to achieve carrier aggregation application by the digital circuit according to the characteristics of $230 \mathrm{MHz}$ band. The transmission rate, spectrum efficiency calculations and analysis results show that the carrier aggregation technology can effectively improve the spectrum efficiency, rational use of $230 \mathrm{MHz}$ spectrum resources.
\end{abstract}

\section{Introduction}

Smart grid is bi-network including the power grid and information \& communication network, which adopts the communication, network, advanced sensing and measurement technologies, the advanced set preparation technology, advanced control methods and advanced decision support technology etc[1]. Especially, in the power distribution and utilization, the optical fiber communication network coverage is limited. Therefore, the wireless technology is conducive to transmission and distribution of electrical equipment, lines, tower and other intelligent monitoring anti- protection, real-time dynamic smart metering, smart grid and user interaction.

However, in $223 \sim 231 \mathrm{MHz}$ wireless band, the power system is just allocated 10 simplex frequency and 15 pairs of frequency duplex transceiver interval of $7 \mathrm{MHz}$, and the frequency bandwidth is small (only a few dozen $\mathrm{kHz}$ ), which is unable to meet the intellectual smart grid high bandwidth communications needs. Meanwhile, the mobile communication applications in smart grad is growing rapidly, especially multimedia services, video-on-demand business applications, which needs higher peak rates, greater bandwidth spectrum resources source. Therefore, 3GPP evolution of 3G systems proposed plan: LTE-Advanced (Long Term Evolution), and provides LTE-A system, key technical indicators [2]. 3GPP allocates the $450 \sim 470 \mathrm{MHz}, 698 \sim 862 \mathrm{MHz}$. $790 \sim 862 \mathrm{MHz}, 2.3 \sim$ 2.4GHz, $3.4 \sim 4.2 \mathrm{GHz}$, and4.4 $\sim 4.99 \mathrm{GHz}$ frequency bands to LTE- A, and requires a maximum spectral bandwidth to $100 \mathrm{MHz}$. However, in practical engineering, such a wide continuous spectrum is difficult to find, thus the LTE-A proposed the carrier aggregation technology [3].

In this paper, we study the electrical communication with integrated access technology to establish communication with the whole business model of electricity based on the same frequency and carrier aggregation TD-LTE230 MHz power wireless broadband system. The carrier aggregation (CA) is a plurality of discrete or continuous aggregation a small bandwidth bands to form a wider frequency 
band and support higher bandwidth data transmission. Each band is referred to as polymeric component carrier (CC) which is called carrier frequency [5]. Distributed in different ways according to the component carrier, carrier aggregation can be divided into intraband contiguous CA, intraband non-contiguous CA as well as non-contiguous CA as shown in fig 1.

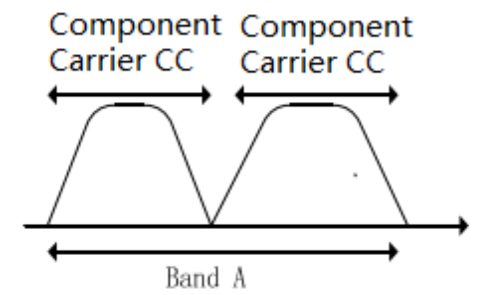

(a) Intraband contiguous $\mathrm{CA}$

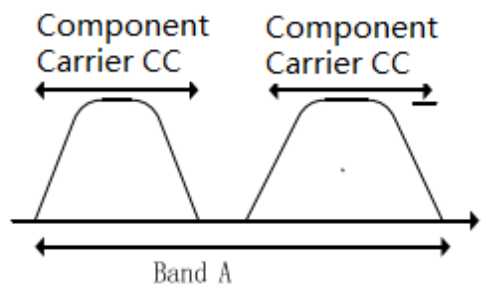

(b) Intraband noncontiguous CA

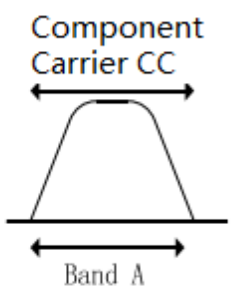

(c) Interband noncontiguous CA

Fig 1. The carrier spectrum aggregation type

The rest of this paper is organized as follows. Firstly, we introduce carrier aggregation and to improve the spectral efficiency of this paper. And then, we introduce adaptive modulation and coding (AMC) technology and analyze the coding rate. Finally, we give the summary

\section{Carrier Aggregation (CA)}

Carrier aggregation is very suitable for solving the discrete spectrum of high-speed transmission and application in $230 \mathrm{MHz}$ wireless access to improve the data transfer capability. When the narrowband spectrum is discrete, each discrete channel can be seen a member of the carrier. Meanwhile, it aggregates the carrier members discontinuous and uniform distribution to a user, which can produce several times more than the original narrow-band system transmission bandwidth to achieve broadband transmission in combination with other communications technology and other higher order modulation as shown in fig 2.

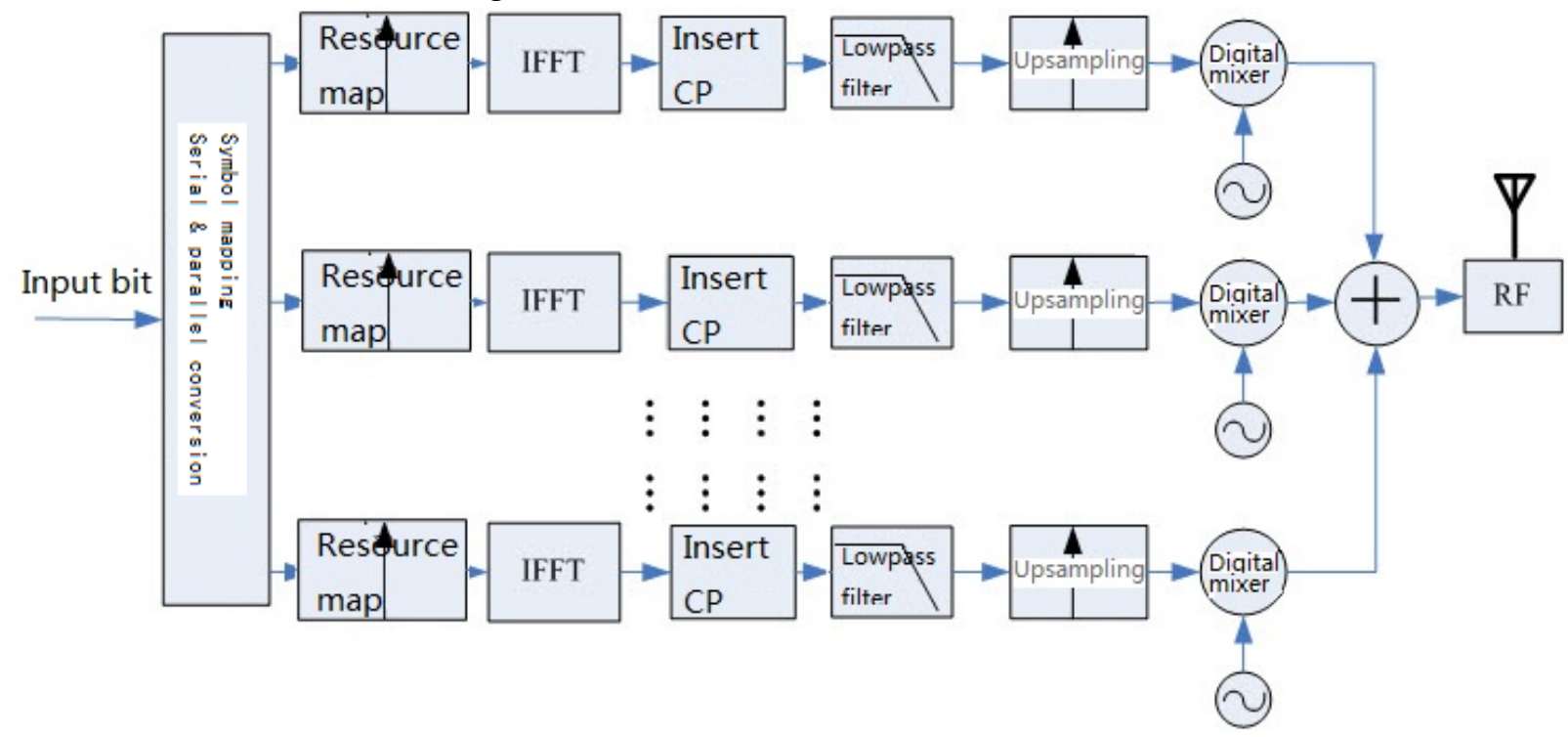

Fig 2. The Carrier Aggregation Mechanism

With the digital intermediate frequency (IF) technology development, the entire aggregation process can be implemented IF by digital circuitry which makes hardware costs under control and improve the reliability. Carrier aggregation method can effectively integrate the limited spectrum resources, improve spectral efficiency, and be suitable for achieving higher spectral efficiency in the limited spectrum resources dedicated power to overcome the current radio transmission rate is low, real poor, spectrum efficiency low shortcomings. 
According to the characteristics of power frequency spectrum, we divide the bandwidth into multiple sub-bands, every sub-band is $25 \mathrm{kHz}$ (total 40 sub-bands). However, for protecting the bandwidth, we define each sub-band as $22 \mathrm{kHz}$. Assume $\Delta s$ is the interval between the adjacent sub-band. Then we can get the number of sub-band in the system is as $\mathrm{Eq}(1)$ :

$$
N_{\text {subband }}=\left(\frac{22000}{\Delta S}\right) \times 40
$$

The number of symbol in each slot is as Eq (2):

$$
N_{\text {symbol }}=\left(\frac{0.5}{T_{\text {symbol }}}\right) \times 2
$$

Where the $N_{\text {subband }}$ is the number of sub-band; $N_{\text {symbol }}$ denotes the number of symbol in each solt; and $T_{\text {symbol }}$ is the symbol period. According to Eq (1) and (2), we can calculate the sub-band levels of power wireless broadband communication system terminal as shown in Table 1 ..

Table 1. The sub-band levels of power wireless broadband communication system terminal

\begin{tabular}{cccc}
\hline Terminal level & Bandwidth & Uplink peak rate & $\begin{array}{c}\text { Downlink peak } \\
\text { rate }\end{array}$ \\
\hline Single subband & $25 \mathrm{kHz}$ & $44 \mathrm{kbps}$ & $19.8 \mathrm{kbps}$ \\
10 subbands & $250 \mathrm{kHz}$ & $396 \mathrm{kbps}$ & $178.2 \mathrm{kbps}$ \\
20 subbands & $500 \mathrm{kHz}$ & $836 \mathrm{kbps}$ & $376.2 \mathrm{kbps}$ \\
40 subbands & $1 \mathrm{MHz}$ & $1.7 \mathrm{Mbps}$ & $772.2 \mathrm{kbps}$ \\
\hline
\end{tabular}

\section{Adaptive modulation and coding (AMC)}

Using the adaptive modulation and coding (AMC) technology, we can employ different coding scheme to meet the requirement of data transfer speed in different environment. The lower order modulation can tolerate higher intensity interference but the lower the transmission bit rate; the higher order modulation may offer higher bit rate better in channel conditions[4], such as the QPSK (423.4kbit/s), 16QAM(939.8kbit/s) and 64QAM(1760.0kbit/s) etc. Moreover, to improve the system ability to quickly and efficiently respond to changes in the channel, the link performance and maximize system capacity, we can employ the Hybrid Automatic Repeat reQuest technology (HARQ) [6].

HARQ is a technique combined the forward error correction (FEC) and automatic repeat request ARQ. Based on the function that the channel conditions can provide more accurate rate adjustment, HARQ can automatically adapt to the instantaneous channel conditions which is less affected by transient channel measurement results and delay. HARQ provides fine data rate adjustment based on channel conditions. HARQ technology is mainly implemented in two ways: one is in the retransmission, the retransmission data with the same initial retransmission, which is called tracking merger (CC) or soft merger; the other is a retransmission initial data transmission time is different, and in this way is called incremental redundancy (IR). IR is divided into partial incremental redundancy (PIR) and full incremental redundancy (FIR).

When using 16QAM modulation, the second retransmission power is equal to the first. When the transport channel type is PB3, we adopt coding rate $1 / 2$ and $3 / 4$, respectively, we can obtain the results as shown in fig 3 , which show the relationship between BLER and Ior / Ioc. 

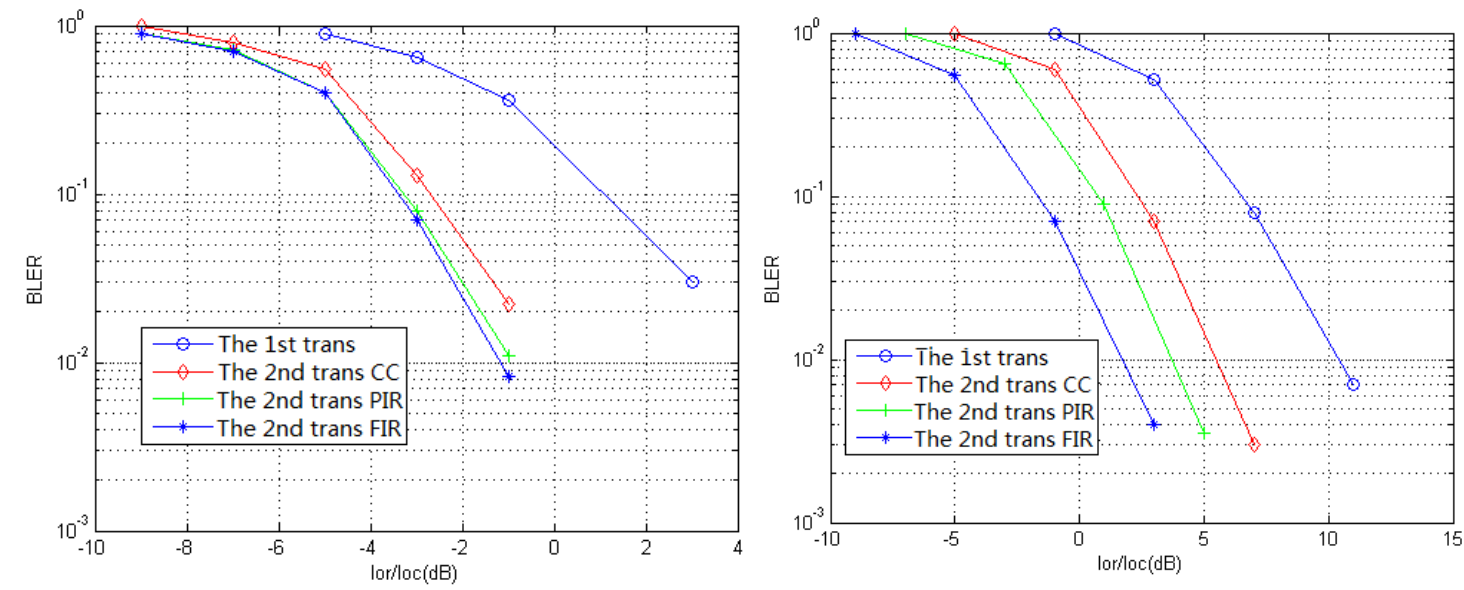

Fig 3. The coding rate is $1 / 2$ and $3 / 4$

In $10 \%$ of cases BLER, PIR way better than the CRC about $2 \mathrm{~dB}$, because PIR ways to pass additional check bits; FIR PIR better way than $3 \mathrm{~dB}$, this is because when the coding rate is high, FIR can pass than PIR more parity bits.

\section{Summary}

In the power distribution and utilization, the optical fiber communication network coverage is limited, and the wireless technology is usually adapted to meet the requirement of transmission and distribution of electrical applications. In our solution, the smart grid and user interaction improve the efficiency of the power spectrum dedicated $230 \mathrm{MHz}$ spectrum proposed the 40 discrete frequency bands of the carrier aggregation thoughts. According to the characteristics of $230 \mathrm{MHz}$ band, we present a novel scheme to achieve carrier aggregation application by the digital circuit. The transmission rate, spectrum efficiency calculations and analysis results show that the carrier aggregation technology can effectively improve the spectrum efficiency, rational use of $230 \mathrm{MHz}$ spectrum resources.

\section{References}

[1] X.Y Liu, L.H Wang, D.D Liu et al. Energy-efficient algorithm for carrier aggregation and cognitive radio based TD-LTE system, Information and Communications Technologies (ICT 2014), 2014 International Conference on. IET, 2014:1-5 .

[2] 3GPP TR 36.913 v8.0.0. Requirements for Further Advancements for E-UTRA (LTEAdvanced).

[3] 3GPP REV-080030, Technology components, ERICSSON, April, 7-8, 2008.

[4] J.P Cao, J.M Liu, X.Z Li, Carrier Aggregation Technology on 230MHz Dedicated Spectrum of Power Systems, Automation of Electric Power Systems, Vol (37), 2013 (in Chinese)

[5] Q Zhang, L Guo, C Sun et al. Joint power control and component carrier assignment scheme in heterogeneous network with carrier aggregation, Iet Communications, 2014, 8(10):1831-1836.

[6] T Breddermann, B Eschbach, P Vary. On the Design of Hybrid Automatic Repeat Request Schemes with Unreliable Feedback. Communications IEEE Transactions on, 2014, 62(62):758-768. 